Chirurg 2020 $91: 978$

https://doi.org/10.1007/s00104-020-01296-3 (c) Springer Medizin Verlag GmbH, ein Teil von Springer Nature 2020

\section{Redaktion}

Prof. W. Schröder, Köln

Ein Kooperationsprojekt mit dem BDC und der DGCH

In Kürze findet ein Webinar statt, basierend auf der Leitlinie ,Interdisziplinär abgestimmte Empfehlungen zum Personal- und Patientenschutz bei Durchführung planbarer Eingriffe zur Zeit der SARS-CoV-2-Pandemie", wozu der Berufsverband der Deutschen Chirurgen e.V. (BDC), die Deutsche Gesellschaft für Chirurgie (DGCH) und Springer Medizin einladen.

Die Wiederaufnahme planbarer Aufnahmen und Operationen sollte stufenweise mit Blick auf die Vorhaltung notwendiger Intensivkapazitäten für COVID-19-Patienten erfolgen und wird aktuell bundesweit umgesetzt.

- Die Leitlinie adressiert das einzugrenzende Patientenrisiko durch Früherkennung bzw. Minimierung von SARS-CoV-2-Infektionen bei elektiven chirurgischen/operativen $\mathrm{Pa}$ tienten und den (dafür notwendigen) Schutz des behandelnden medizinischen Personals:

\footnotetext{
In den Leitlinien wird das aktuelle Wissen zu chirurgisch relevanten Themen nach Zusammenstellung und Auswertung der vorhandenen Literatur entsprechend evidenzbasierter Kriterien zusammengefasst. Die Verfasser dieser Rubrik weisen jedoch darauf hin, dass es zu vielen Empfehlungen der Leitlinie keine wissenschaftliche Evidenz im eigentlichen Sinne gibt, sondern diese oftmals auf einem Expertenkonsens der verschiedenen Fachdisziplinen beruhen. Somit können Empfehlungen der Leitlinien keine rechtlich bindenden Anweisungen für ärztliches Handeln darstellen, sondern bieten in Diagnostik und Therapie eine Hilfestellung für den ärztlichen Alltag.
}

Andreas Dietz $\cdot$ Hans-Joachim Meyer

Klinik und Poliklinik für Hals-, Nasen-, Ohrenheilkunde, Department Kopf- und Zahnmedizin, Universitätsklinikum Leipzig, Leipzig

\title{
Webinar zur S1-Leitlinie
}

\section{„Interdisziplinär abgestimmte Empfehlungen zum Personal- und Patientenschutz bei Durchführung planbarer Eingriffe zur Zeit der SARS-CoV-2-Pandemie"}

- Wie sinnvoll ist die präoperative SARS-CoV-2-PCR(„polymerase chain reaction")-Abstrichtestung?

- Gibt es bei der präoperativen Abstrichtestung bei Kindern Besonderheiten $\mathrm{zu}$ beachten?

- Gelten für Antikörpertestungen die gleichen Bedingungen wie für präoperative Abstrichtestungen?

- Welche persönliche Schutzausrüstung wird empfohlen?

- Was ändert sich in der Planung bei planbaren Eingriffen im Vergleich zu früher?

- Über was sollte der Patient zusätzlich vor der Operation aufgeklärt werden?

\section{Allgemeine Angaben zur Leitlinie}

- Organisation: Prof. Dr. Andreas Dietz

- Führende Fachgesellschaft:

Deutsche Gesellschaft für Hals-Nasen-Ohren-Heilkunde, Kopf- und Hals-Chirurgie e. V. (DGHNO-KHC)
Deutsche Gesellschaft für Chirurgie e. V. (DGCH)

- Registrierung: 017 - 080

- Veröffentlichung: 03.06.2020

- Gültigkeitsdauer: 03.06.2021

- Publikation/Download: https://www. awmf.org/leitlinien/detail/ll/017-080. html

\section{Korrespondenzadresse}

\section{Andreas Dietz}

Klinik und Poliklinik für Hals-, Nasen-, Ohrenheilkunde, Department Kopf- und Zahnmedizin, Universitätsklinikum Leipzig

Liebigstraße 10-14, 04103 Leipzig,

Deutschland

andreas.dietz@medizin.uni-leipzig.de

Interessenkonflikt. A. Dietz und H.-J. Meyer geben an, dass keine Interessenkonflikt besteht.
Infobox Live-Webinar: Save the date

- Termin: Donnerstag, 19. November 2020, 18.00 bis $19.00 \mathrm{Uhr}$

- Login: www.bdc.de (über Mitgliedsnummer BDC/DGCH) Referenten: Prof. Dr. med. Andreas Dietz

- Dieses und alle weiteren Webinare finden Sie einen Tag nach der Liveveranstaltung in den folgenden Archiven: www.bdc-webinare.de und www.SpringerMedizin.de

- Achtung: Dieses Webinar wird aufgrund der Kurzfristigkeit NICHT CME-zertifiziert werden!

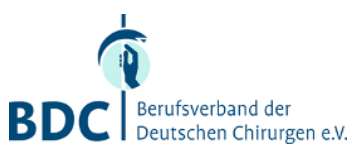

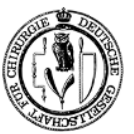

Springer Medizin 